\title{
The spermidine analogue GC7 (N1-guanyl-1,7-diamineoheptane) induces autophagy through a mechanism not involving the hypusination of eIF5A
}

\author{
Serafina Oliverio $\cdot$ Marco Corazzari $\cdot$ Claudia Sestito $\cdot$ \\ Lucia Piredda · Giuseppe Ippolito $\cdot$ Mauro Piacentini
}

Received: 19 May 2014 / Accepted: 31 July 2014

(C) Springer-Verlag Wien 2014

\begin{abstract}
The exogenous administration of spermidine promotes longevity in many model organisms. It has been proposed that this anti-age activity of spermidine is related to this polyamine's ability to promote autophagy. Since spermidine is the substrate for the eIF5A post-translational modification by hypusination, we asked ourselves whether mature eIF5A may represent the link between spermidine and autophagy induction. To test this hypothesis, we inhibited the conversion of native eIF5A by a pharmacological approach, using the N1-guanyl-1,7-diamineoheptane (GC7), a spermidine analogue which competitively and reversibly inhibits deoxyhypusine synthase (DHS). In addition, we also employed genetic approaches by ablating both the eIF5A protein itself and DHS, the rate limiting enzyme catalyzing the conversion of lysine to hypusine. Collectively the data presented in this study demonstrate that the mature eIF5A (hypusinated form) is not involved in the autophagic pathway and that the inhibitor of DHS, GC7, produces off-target effect(s) resulting in marked induction of basal autophagy.
\end{abstract}

S. Oliverio and M. Corazzari have equally contributed to this work.

S. Oliverio $\cdot$ M. Corazzari $\cdot$ C. Sestito $\cdot$ L. Piredda $\cdot$

M. Piacentini $(\bowtie)$

Department of Biology, University of Rome 'Tor Vergata',

Via Della Ricerca Scientifica, 00133 Rome, Italy

e-mail: mauro.piacentini@uniroma2.it

M. Corazzari $\cdot$ G. Ippolito $\cdot$ M. Piacentini

National Institute for Infectious Diseases I.R.C.C.S. 'Lazzaro

Spallanzani', 00149 Rome, Italy

C. Sestito

Department of Anatomy and Neurosciences, VU University

Medical Center, Neuroscience Campus, Amsterdam,

The Netherlands
These data are relevant in light of the fact that GC7 is considered a potent and selective inhibitor of DHS and is a potential candidate drug for cancer, diabetes and HIV therapy.

Keywords eIF5A · Autophagy - GC7 · DHS · Cancer · HIV
Abbreviations
eIF5A Eukaryotic initiation factor 5A
DHS Deoxyhypusine synthase
GC7 N1-guanyl-1,7-diaminoheptane

\section{Introduction}

Spermidine is a natural polyamine ubiquitously highly present in all living organisms; it has been implicated in many pathophysiological processes including cellular proliferation, transformation, differentiation, apoptosis, ageing and tumorigenesis (Gerner and Meyskens 2004; Pegg 2009; Igarashi and Kashiwagi 2010; Mandal et al. 2013). The exogenous administration of spermidine promotes longevity in many model organisms including yeast, nematodes and flies, and significantly reduces age-related oxidative protein damage in mice (Eisenberg et al. 2009; Madeo et al. 2010; Morselli et al. 2011; Tirupathi et al. 2011). It has been postulated that the anti-age activity of spermidine could be related to this molecule's ability to modulate the autophagic process (Eisenberg et al. 2009). Of note, spermidine plays a pivotal role in the post-translational modification of the eukaryotic initiation factor 5A (eIF5A), consisting in protein hypusination (Huang et al. 2007). eIF5A is a small (17 kDa) acidic protein carrying a unique polyamine-derived amino acid, hypusine [Ne-(4-amino-2-hydroxybutyl)lysine] (Caraglia et al. 2013; Shiba et al. 1971; Cooper et al. 1982). 
Fig. 1 Schematic representation of eIF5A post-translational modification. Deoxyhypusine synthase (DHS) catalyzes the transfer of the aminobutyl moiety from spermidine to a specific lysine residue (Lys50 in human eIF5A) to form the deoxyhypusine intermediate, [NE-(4-aminobutyl)-lysine] residue; the intermediate is subsequently hydroxylated by deoxyhypusine hydroxylase (DOHH) to produce active hypusinated eIF5A

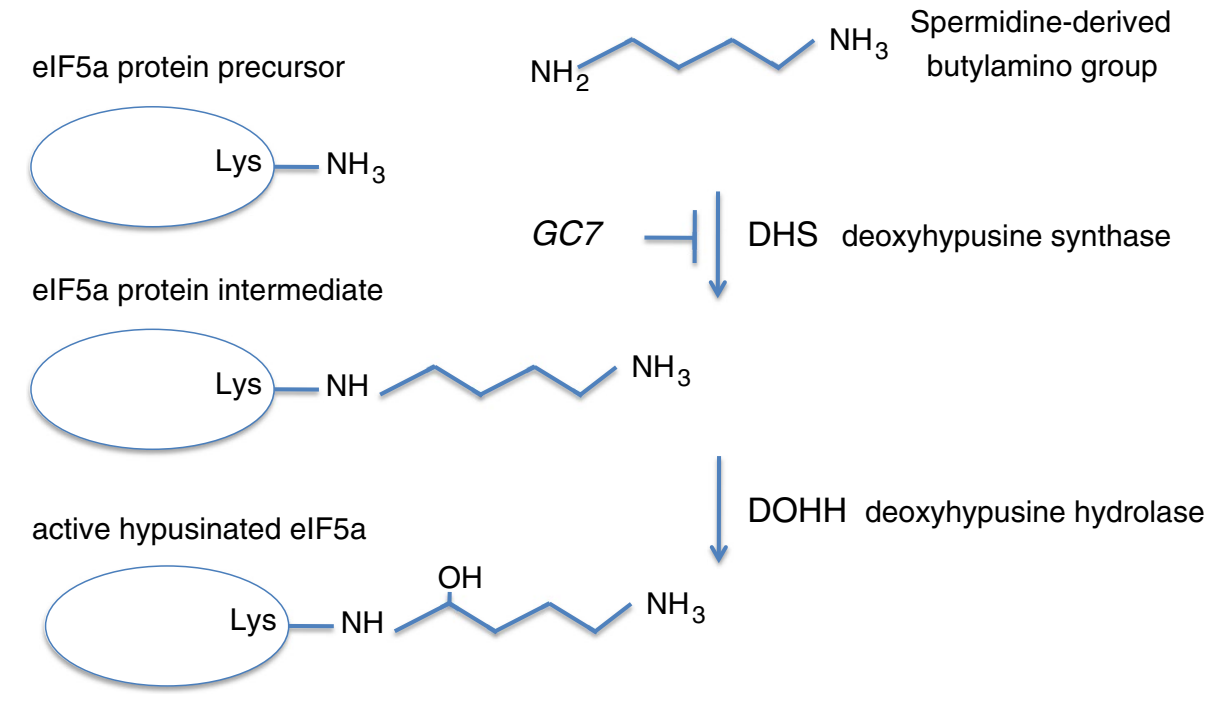

Hypusine is synthesized from the polyamine spermidine in two sequential enzymatic steps: in the first step deoxyhypusine synthase (DHS) catalyzes the transfer of the aminobutyl moiety from spermidine to a specific lysine residue (Lys50 in human eIF5A) to form the deoxyhypusine intermediate, [NE-(4-aminobutyl)-lysine] residue; the intermediate is subsequently hydroxylated by deoxyhypusine hydroxylase (DOHH) to produce active hypusinated eIF5A (Park 2006) (Fig. 1). Two isoforms of eIF5A sharing $84 \%$ homology exist in humans although showing distinct biological functions (Caraglia et al. 2013). eIF5A-1 is ubiquitously expressed and its level is particularly high in proliferating cells; by contrast, eIF5A-2 has a more restricted expression (Jenkins et al. 2001; Guan et al. 2004). There are a lot of evidences to indicate that eIF5A is a key protein in the pathogenicity of different diseases, such as diabetes, several human cancers, malaria and HIV-1 infection (Kaiser 2012).

Although the physiological role of eIF5A-1 has not yet been fully elucidated, it has been found to function: (a) as a translation elongation factor during protein synthesis (Saini et al. 2009), (b) as a cytoplasmic shuttling protein regulating mRNA transport (Liu et al. 1997; Maier et al. 2010) and (c) as a cellular cofactor of HIV-1 REV (Benne and Hershey 1978). It has also been implicated in the regulation of mRNA turnover (Zuk and Jacobson 1998), cell proliferation (Park et al. 1993, 2010), differentiation (Schnier et al. 1991; Park et al. 2010), inflammation, (Moore et al. 2008) and apoptosis (Taylor et al. 2013). Interestingly, the pro-apoptotic function of eIF5A-1 appears to be the only eIF5A activity which is independent from hypusine modification (Taylor et al. 2007, 2012; Sun et al. 2010). Growing evidence indicates that apoptosis induction is often associated with decreased autophagy, underlying the existence of an interplay between these two important cellular events (Fimia and Piacentini 2010).
Autophagy is an intracellular degradation system which delivers cytoplasmic constituents to the lysosome (Xie and Klionsky 2007). This is a highly conserved process in eukaryotes and has two main physiological functions: it removes unwanted/aged/damaged constituents and recycles cytoplasmic materials to maintain macromolecular synthesis and energy homeostasis during stressful conditions including nutrient deprivation, hypoxia and low energy status.

Although Patel et al. (2009) have hypothesized that the Drosophila deoxyhypusine hydroxylase homologue Nero and its target eIF5A are involved in autophagy regulation, no direct evidence shows eIF5A's involvement in the autophagic process, at least until now. On the other end, what has been recently demonstrated is spermidine's ability to stimulate autophagy in yeast, nematodes and flies, increasing the overall lifespan (Morselli et al. 2011), albeit the molecular mechanism is still unclear.

Therefore, since spermidine is directly required for eIF5A modification by hypusination, we asked whether mature eIF5A may represent the link between spermidine and autophagy. To test this hypothesis, we inhibited the conversion of native eIF5A by both pharmacological and genetic approaches and evaluated the impact on autophagy. Here we show that GC7 has an off-target effect, since its administration results in cell basal autophagy induction, independently of eIF5A activity.

\section{Materials and methods}

\section{Materials}

Mouse Anti-eIF-5a (BD 611976; dilution 1:10,000) was from BD Biosciences; rabbit anti-DHS (sc-67161; dilution 1:1,000) and mouse anti-Gapdh (sc-47724; dilution 
1:1,000) were from Santa Cruz Biotechnology; rabbit antiLC3 (NB100-2331; dilution 1:500) and Rat anti-FLAG (dilution 1:500) were from Novus Biologicals.

The anti-mouse or anti-rabbit secondary antibodies HRP-conjugated (dilution 1:5,000) and the ECL detection system (Immun-StarTM WesternTM Kit) were from BioRad. Earle's balanced salt solution (EBSS), Bafilomycin A1, chloroquine, E64D and Pepstatin A were obtained from Sigma. N1-Guanyl-1,7-diaminoheptane (GC7) was from Biosearch Technologies; D-MEM and FBS were from Invitrogen Life Technologies.

\section{Cell culture and treatments}

Human fibrosarcoma 2fTGH cells (2F) were cultured in Dulbecco's modified Eagle's medium (D-MEM) supplemented with $10 \%$ foetal bovine serum, $2 \mathrm{mM}$ L-glutamine, $100 \mathrm{mg} /$ $\mathrm{ml}$ streptomycin and 100 units/ml penicillin. Cells were grown in a humidified atmosphere containing $5 \% \mathrm{CO}_{2}$ at $37^{\circ} \mathrm{C}$.

$2 \times 10^{5}$ cells were treated with $200 \mu \mathrm{M} \mathrm{GC7}$ (dissolved in $10 \mathrm{mM}$ of acetic acid), $10 \mathrm{nM}$ Bafilomycin A1 (BafA), $10 \mu \mathrm{g} / \mathrm{ml}$ of both E64d and Pepstatin A, as indicated. All compounds were dissolved in DMSO except GC7 that was dissolved in $10 \mathrm{mM}$ acetic acid.

\section{Western blotting}

Cells were rinsed in ice-cold phosphate-buffered saline (PBS) and collected in lysis buffer (20 mM Tris- $\mathrm{HCl}$ pH 7.4, $150 \mathrm{mM} \mathrm{NaCl}, 1 \%$ Triton X-100) plus protease and phosphatase inhibitors (protease inhibitor cocktail, $1 \mathrm{mM}$ sodium fluoride, $1 \mathrm{mM}$ sodium orthovanadate, Sigma). Samples were centrifuged $15 \mathrm{~min}$ at $9,000 \times g$ and, total protein concentration was evaluated by the DC protein Kit (Bio-Rad).

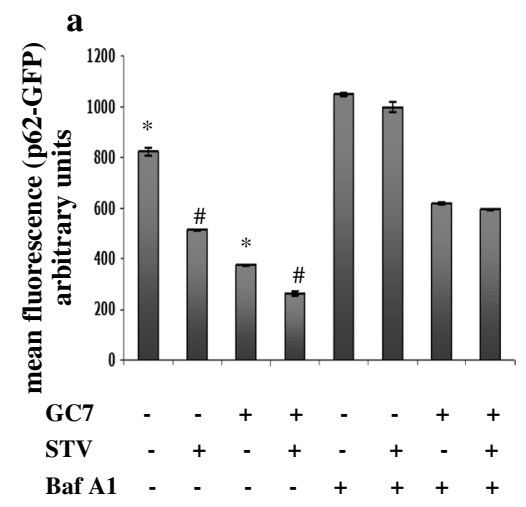

b
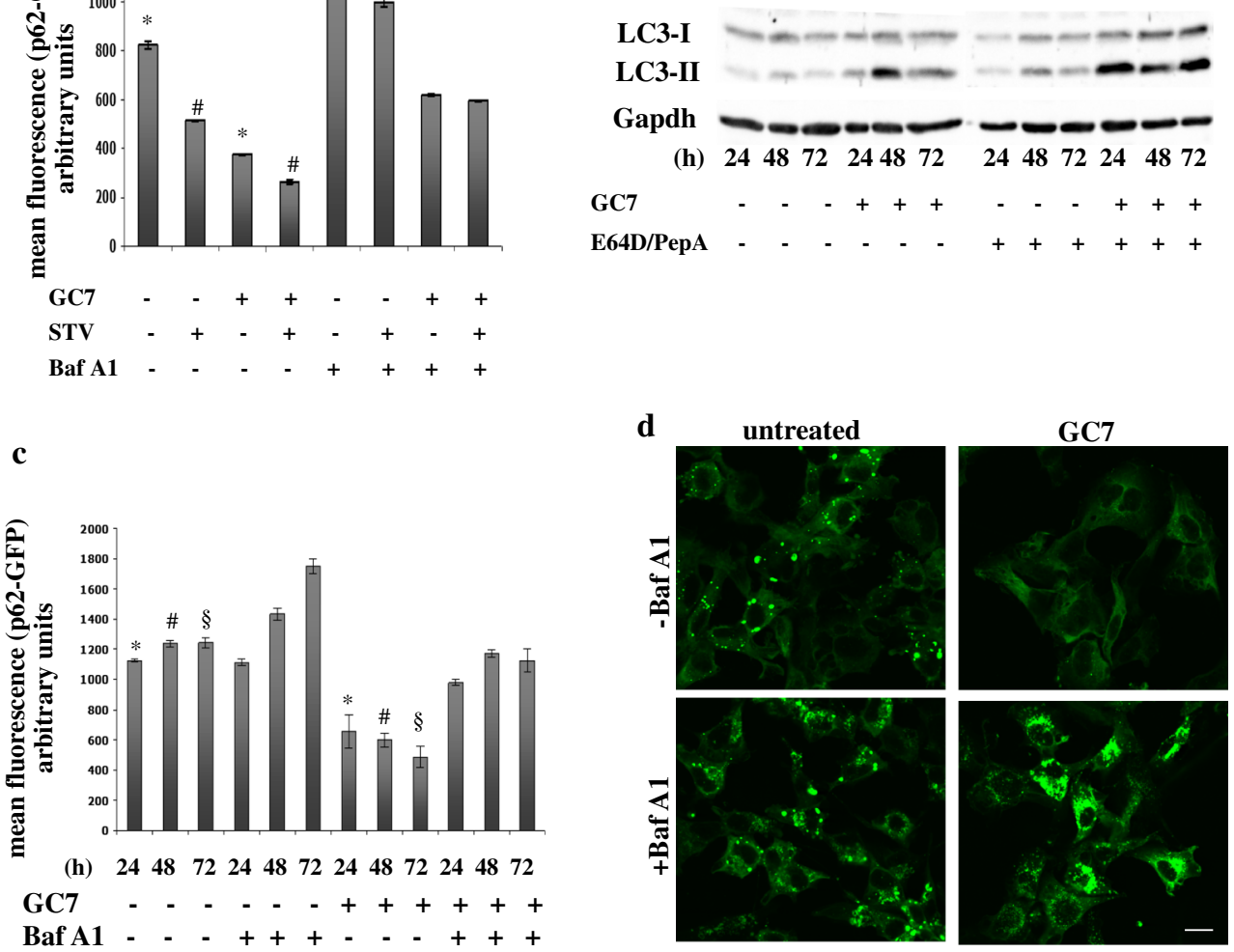

Fig. 2 Autophagy induction by GC7, an eIF5A inhibitor. a 2F cells stably expressing a p62-GFP recombinant protein were grown in the presence or absence of $200 \mu \mathrm{M} \mathrm{GC7}(18 \mathrm{~h})$ and treated or untreated with $10 \mathrm{nM}$ Bafilomycin A1 (6 h) in normal or EBSS medium. p62 degradation was evaluated by flow cytometry as the mean of fluorescence $\pm \mathrm{SD}$ of three independent experiments $(*, \# p<0.05)$. b $2 \mathrm{~F}$ cells were treated for $24,48,72 \mathrm{~h}$ with $200 \mu \mathrm{M} \mathrm{GC7}$ and in the presence or absence of $10 \mu \mathrm{g} / \mathrm{ml} \mathrm{E64D/Pepstatin} \mathrm{A} \mathrm{(the} \mathrm{last} 6 \mathrm{~h}$ ). LC3 conversion was determined by Western blotting analysis. Gapdh was

used as a loading control. c $2 \mathrm{~F}$ cells stably expressing a p62-GFP recombinant protein were treated or untreated with $\mathrm{GC7}$, as indicated, in the presence or absence of Bafilomycin A1, and p62 degradation was evaluated by flow cytometry as the mean of fluorescence \pm SD of three independent experiments $\left(*, \#,{ }_{p}<0.05\right)$. d Representative fluorescent micrographs of $2 \mathrm{~F}$ cells stably expressing a p62-GFP recombinant protein treated or untreated $24 \mathrm{~h}$ with GC7 and/or Bafilomycin A1. Bar $10 \mu \mathrm{m}$ 
Proteins were resolved by a $12 \%$ SDS-PAGE and transferred onto a nitrocellulose membrane. Membranes incubated $1 \mathrm{~h}$ with $5 \%$ nonfat dry milk in T-PBS containing $0.05 \%$ Tween $20(1 \mathrm{~h})$ and then incubated overnight with indicated antibodies, at $4{ }^{\circ} \mathrm{C}$. After three washes with T-PBS, membranes were incubated $1 \mathrm{~h}$ with HRP-conjugated secondary antibody, at rt. Membranes were rinsed three times with T-PBS, and the signal was detected by enhanced ECL Immunostar detection system from BioRad.

\section{Retroviral expression of GFP-p62 and mRFP-GFP-LC3}

Fifteen microgram of retroviral vectors (GFP-p62 or RFPGFP-LC3) was co-transfected with $5 \mu \mathrm{g}$ of an expression plasmid for the vesicular stomatitis virus $G$ protein into $293 \mathrm{gp} / \mathrm{bsr}$ cells using the calcium phosphate method. After $48 \mathrm{~h}$, the supernatant containing the retroviral particles was recovered and supplemented with polybrene $(4 \mathrm{mg} / \mathrm{mL})$. $2 \mathrm{~F}$ cells were infected by incubation with retroviral-containing supernatant for 6-8 h, as previously described (Pagliarini et al. 2012).

\section{Autophagy analysis}

For confocal microscopy analysis, $2 \times 10^{5}$ cells were grown on glass cover slips, fixed using $4 \%$ paraformaldehyde, and fluorescence analyzed by a Leica TCS SPII laser-scanning confocal microscope, as previously reported (Hill et al. 2009).

p62-GFP flow cytometric analysis was performed by monitoring the green-fluorescence intensity of p62 protein. Briefly, 2fp62GFP cells were fixed by $4 \%$ paraformaldehyde, 20,000 events were acquired by a FACScan cytometer (Becton-Dickinson) and data analyzed using CellQuest software (Pagliarini et al. 2012).

\section{RNA interference}

RNAi was performed using the following oligonucleotides from Ambion:

Oligos a-1 or b-1 were eIF5A Silencer Selected Predesigned siRNA \# 4392420 or Custom Selected siRNA \#4390827 for eIF5A-1; siRNA \#4390824 for DHS and $\# 12935-300$ as a negative control (siCtrl).

$5 \times 10^{5}$ cells/well were transfected with 100 pmol siRNA in a six-well plates using lipofectamine RNAimax (Invitrogen), as indicated by the supplier. Transfection was blocked after $24 \mathrm{~h}$ and cells treated as indicated.

\section{qRT-PCR}

RNA was extracted using Trizol reagent (Invitrogen) as indicated by the supplier. cDNA synthesis was generated using a reverse-transcription kit (Promega, Madison, WI, USA) according to the manufacturer's recommendations. Quantitative PCRs were performed with the Rotor-Gene 6000 (Corbett Research Ltd) thermocycler. Primer sets for all amplicons were designed using the Primer-Express 1.0 software system (Roche):

\section{L34 forward: 5'-GTCCCGAACCCCTGGTAATAGA-3' L34 reverse: $5^{\prime}$-GGCCCTGCTGACATGTTTCTT-3' DHS forward: 5'-GTGTAAAGTGGACGCCTTCTA-3'; DHS reverse: 5'-ACACAGGGATGTGGTTCTTC-3';}

L34 mRNA level was used as an internal control and results were expressed as previously described (Pagliarini et al. 2012).

\section{K50A eIF5A mutant}

p3XFLAG-CMV-10.1 encoding for human eIF5A-1 was kindly provided by Myung Hee Park National Institute of Dental and Craniofacial Research "NIDCR" Bethesda, MD (Clement et al. 2006). The mutant K50A was obtained in our lab using Quick Change Site-Directed Mutagenesis Kit (Stratagene). The primers used for mutate of the plasmid p3XFLAG-eIF5A-1 in the Lysine50 (p3XFLAG-K50A) were:

\section{eIF5A K50A 5'-CTTCGAAGACTGGCGCGCACGGCC ACGCCA-3' \\ eIF5A K50A antisense 5'-TGGCGTGGCCGTGCGCG CCAGTCTTCGAAG-3'}

p3XFLAG-eIF5A-1 was used as mutagenesis template.

PCR amplification products were treated with restriction enzyme DpnI (Fermentas). An aliquot of $5 \mu$ l above PCR product was transformed into DH5a competent cells and inoculated on Luria-Beltrani (LB) plate containing $100 \mu \mathrm{g} / \mathrm{ml}$ ampicillin. A total of ten colonies were selected and their plasmids were isolated by mini-prep. The positive mutants were selected by DNA sequencing.

The wt and the mutant K50A of eIF5A were transiently transfected using lipofectamine LTX as indicated by the supplier.

Statistical analysis

All experiments were performed in replicate and repeated three times. Results were expressed as mean \pm SD of three experiments. Data were analyzed by the $t$ Student test and differences were considered significant when $p<0.05$.

\section{Results}

The inhibitor of eIF5A activity, GC7, increases basal autophagy

To elucidate the role of eIF5A in the autophagic pathway we used the fibrosarcoma cell line $2 \mathrm{fTGH}(2 \mathrm{~F})$ as a model 
and N1-guanyl-1,7-diamineoheptane (GC7), a spermidine analogue that competitively and reversibly inhibits deoxyhypusine synthase (DHS; Lee and Folk 1998; Park et al. 1993; Shi et al. 1996), to inhibit eIF5A hypusination. GC7 is commonly used to block the first step of hypusination of eIF5A, resulting in the accumulation of the native protein (Landau et al. 2010). Characteristic features of early and late stages of autophagy were used to measure autophagy in $2 \mathrm{~F}$ cells, such as the conversion of unconjugated LC3 (LC3-I) to the lipidated form (LC3-II), and the degradation of p62. First, we analyzed the effect of the inhibition of eIF5A hypusination on basal or induced autophagy. To this end, autophagy was stimulated using EBSS medium (starvation, stv) in $2 \mathrm{~F}$ cells stably expressing a p62-GFP recombinant protein (2F-p62GFP cell line); cells were treated or untreated $18 \mathrm{~h}$ with $200 \mu \mathrm{M} \mathrm{GC7}$ in the presence or absence of $10 \mathrm{nM}$ Bafilomycin A1 (BafA1), and autophagy was evaluated by measuring the degradation of p62-GFP protein, by flow cytometry. As reported in Fig. 2a, we observed that the inhibition of eIF5A hypusination leads to a drastic degradation of p62 in cells treated with GC7 alone. This potent GC7 pro-autophagic effect was also able to potentiate the starvation-induced $\mathrm{p} 62$ degradation. The positive effect of GC7 on the autophagic flux was also confirmed by the accumulation of $\mathrm{p} 62$ upon treatment with BafA1. To confirm these findings, $2 \mathrm{~F}$ cells were treated for 24,48 or $72 \mathrm{~h}$ with $200 \mu \mathrm{M} \mathrm{GC7}$ in the presence or absence of $10 \mu \mathrm{g} / \mathrm{ml}$ of both E64D and PepstatinA (PepA), two inhibitors of autolysosome degradation activity, and autophagy induction was evaluated by monitoring the LC3 conversion by Western blotting analysis. As reported in Fig. 2b, GC7 treatment increased basal autophagy in a time-dependent manner as evidenced by the enhanced accumulation of LC3-II in the presence of E64D/PepA. Similar results were obtained in 2Fp62GFP cells in the same experimental conditions, by measuring the degradation of p62-GFP, by flow cytometric analysis (Fig. 2c). To further support these data the p62-GFP degradation was also evaluated by confocal analysis in 2Fp62GFP cells treated or untreated with GC7. This analysis confirmed that the GC7 administration per
Fig. 3 GC7 does not affect the autophagic flux. Representative fluorescent micrographs of $2 \mathrm{~F}$ cells stably expressing an RFP-GFP-LC3 recombinant protein. Cells were grown in the presence of $200 \mu \mathrm{M} \mathrm{GC7}(24 \mathrm{~h})$, vehicle (acetic acid, $24 \mathrm{~h}$ ), EBSS ( $6 \mathrm{~h}$ ) or unconditioned medium. Yellow dots represent immature autophagosomes while red dots represent active autolysosomes. Bar $10 \mu \mathrm{m}$
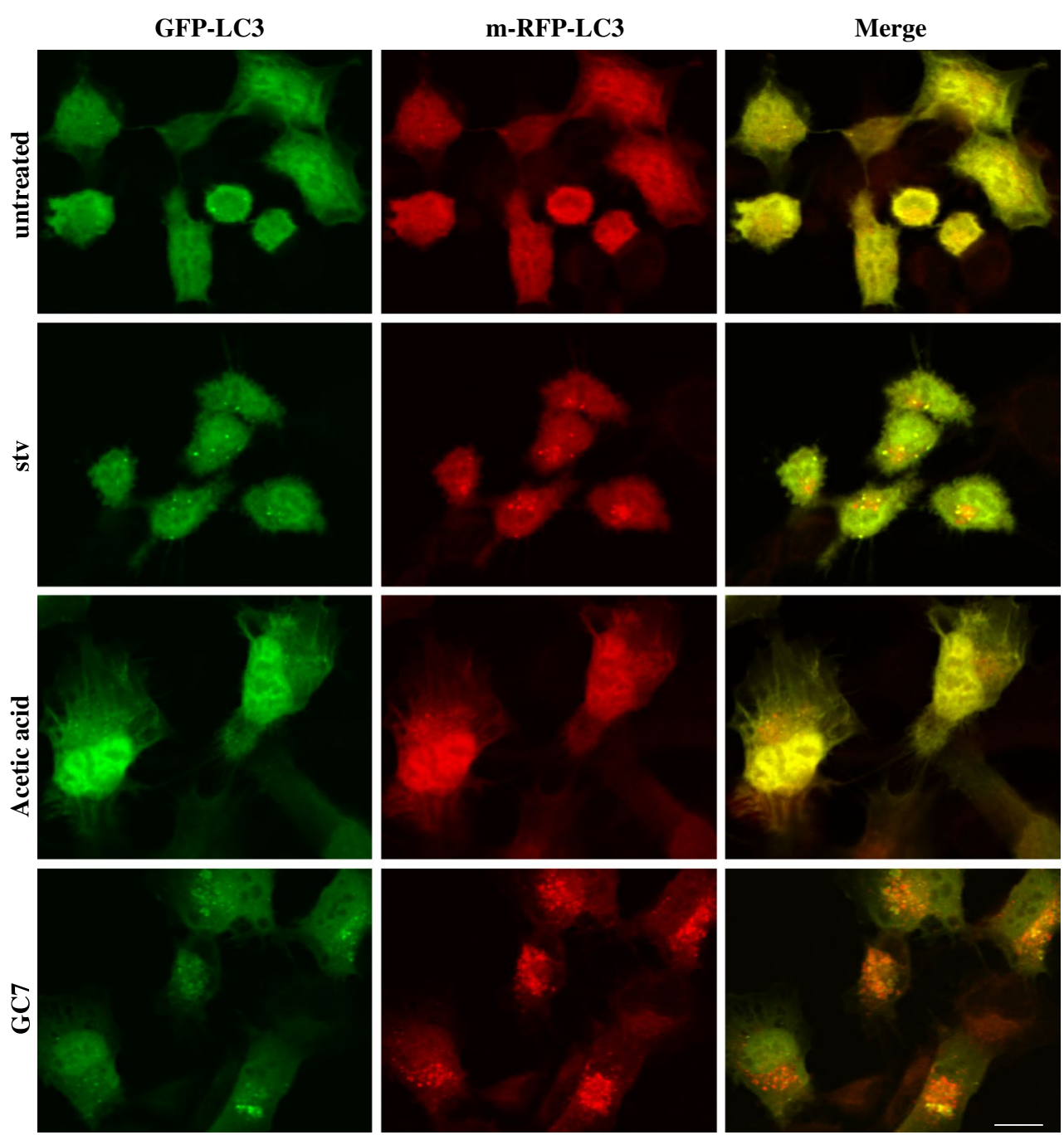

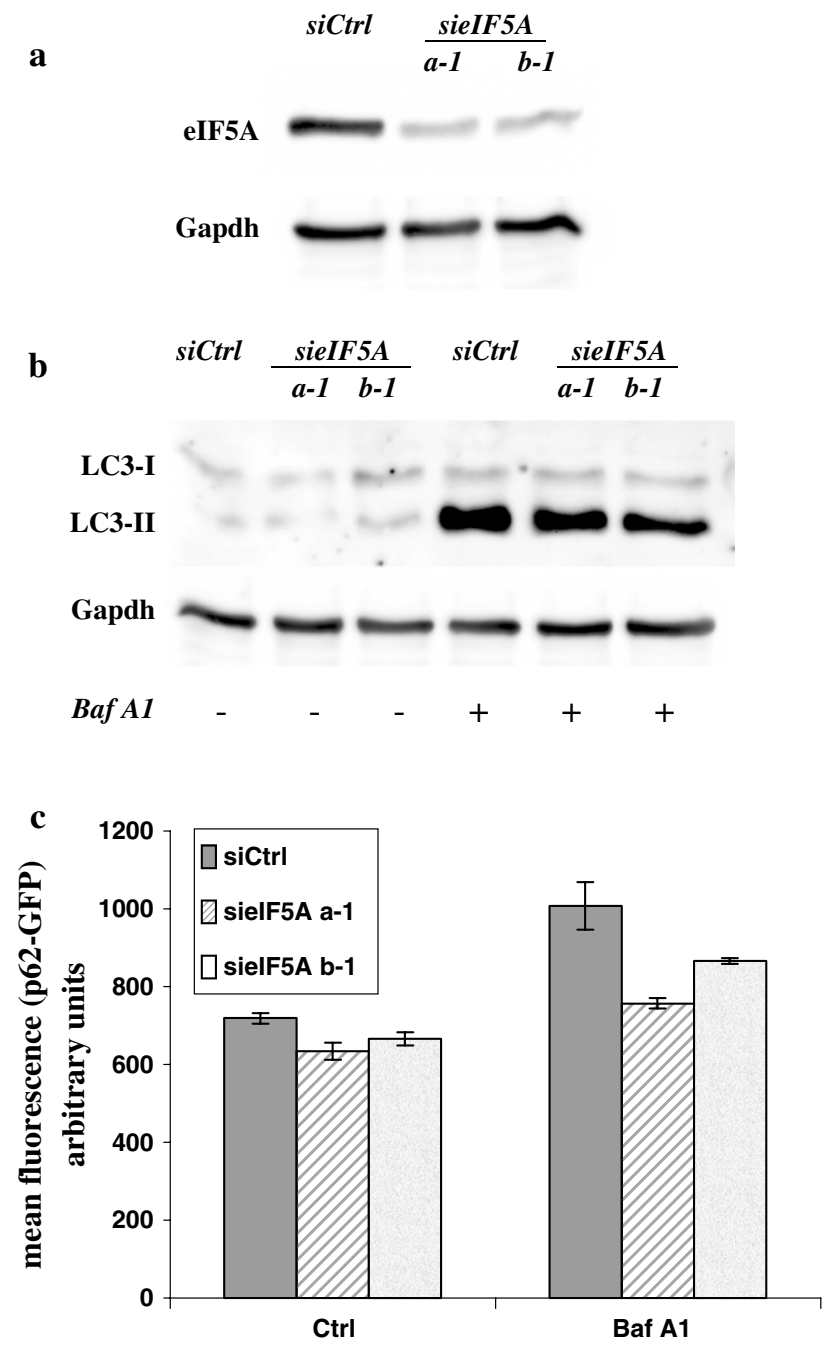

Fig. 4 eIF5A down-regulation does not affect basal autophagy. 2F cells stably expressing a p62-GFP recombinant protein were transiently transfected with two different siRNA oligos specific for eIF5A-1 or with a scramble siRNA (siCtrl) and incubated $6 \mathrm{~h}$ with Bafilomycin A1, as indicated. The expression of eIF5A (a) and the conversion of LC3 (b) were determined by Western blotting analysis, while the degradation of ectopically expressed p62 (c) was evaluated by flow cytometry. Each point represents the mean of fluorescence of p62-GFP protein \pm SD of three different experiments. Gapdh was used as a loading control $(\mathbf{a}, \mathbf{b})$

se resulted in enhanced p62 degradation as evidenced by: (a) decreased GFP fluorescence (p62) compared to control (Fig. 2d, upper right panel compared to upper left panel) and (b) p62-GFP dots cytosolic accumulation in cells treated with GC7 plus BafA1 compared to cells treated with BafA1 alone (Fig. 2d, bottom panels).

Finally, to confirm that the GC7 effect resides in autophagy induction and not in the inhibition of autophagic flux, we used a GFP-RFP-tagged LC3 recombinant protein (Hill et al. 2009). To this end, 2F cells stably expressing GFP-RFP-LC3 were treated with GC7 or vehicle alone
(Acetic Acid; $24 \mathrm{~h}$ ) and autophagy induction was evaluated by confocal analysis. EBSS medium (stv) was used as a positive control. Data reported in Fig. 3 clearly show a dramatic cytosolic accumulation of red-puncta LC3 dots (representing autophagolysosome, mature structures), compared to yellow-puncta ones (representing autophagosome, immature structures), in respect to both vehicle- alone or EBSS-treated cells, indicating a GC7-dependent enhanced complete autophagy induction, but not inhibition of autophagic flux.

\section{eIF5A has no functional role in basal cell autophagy}

To validate by a genetic approach the consistency of our results indicating that eIF5A has a functional role in autophagy, we transiently inhibited the expression of eIF5A-1 (the isoform expressed in 2F cells), using specific siRNA oligos (sieIF5A; Fig. 4a), and cell basal autophagy was evaluated by measuring the conversion of LC3, by Western blotting analysis, in the presence or absence of BafA1 (Fig. 4b).

Although the expression of eIF5A-1 was almost completely inhibited by siRNA oligos (Fig. 4a), the LC3 conversion was only marginally affected, compared to control (siCtrl; Fig. 4b).

Similar results were obtained in 2Fp62GFP cells in the same experimental conditions, by monitoring the degradation of p62-GFP in cells in which the expression of eIF5A-1 was inhibited by siRNA oligos, compared to control (siCtrl; Fig. 4c). These findings suggest that the potent pro-autophagic activity displayed by GC7 was due to an off-target effect not related to the post-translational modification of eIF5A. To support this hypothesis, we decided to verify that also the eIF5A immature form (with no hypusine modification) was also not involved in modulating the autophagic process. To this end, we inhibited the modification of the native eIF5A protein by down-regulating the expression of DHS by the transient transfection of specific siRNA oligo into 2Fp62GFP cells. The inhibition was evaluated by monitoring both eIF5A protein and RNA levels (Fig. 5a). In these cells we analyzed the basal autophagy in the presence or absence of BafA1, by monitoring both the LC 3 conversion by Western blotting analysis (Fig. 5b) and p62 degradation by flow cytometry (Fig 5c). The inhibition of DHS resulted in not statistically significant effects on basal autophagy as evidenced by both LC3-II accumulation and p62 degradation (Fig. 5b, c).

Finally, to confirm that the accumulation of immature eIF5A resulting from inhibition of protein hypusination is not involved in autophagy induction/execution, we ectopically expressed both wild type and K50A mutant eIF5A in $2 \mathrm{~F}$ cells by transient transfection. K50A mutant codes for 
Fig. 5 Inhibiting eIF5A hypusination does not affect $2 \mathrm{~F}$ basal autophagy. a DHS expression was down-regulated in $2 \mathrm{~F}$ cells stably expressing p62-GFP recombinant protein by transient transfection with a specific siRNA (siDHS) and the autophagic flux was inhibited by Bafilomycin A1 treatment $(6 \mathrm{~h})$. A scramble siRNA (siCtrl) was used as a control. The levels of both DHS protein (left panel) and mRNA (right panel) levels were evaluated by Western blotting analysis and qRT-PCR, respectively. b, c LC3 conversion (b) and p62 degradation (c) were determined by Western blotting analysis and flow cytometry, respectively. Gapdh was used as a loading control (a, b). Values are means of fluorescence $\pm \mathrm{SD}$ of three different experiments (c). d $2 \mathrm{~F}$ cells were transiently transfected with a Flag-tagged eIF5A-1 wild type (wt) or a K50A mutant (K50) or with empty vector (Empty) and eIF5A-1 expression and LC3 conversion were evaluated by Western blotting analysis, in the presence or absence of Bafilomycin $(6 \mathrm{~h})$, as indicated

an eIF5A protein in which the hypusination site (Lys50) has been abrogated (replaced with an Ala). As shown in Fig. 5d, the over-expression of both the wt and K50A eIF5A did not affect the basal autophagy of $2 \mathrm{~F}$ cells, thus confirming the off-target effect of GC7 in the induction of autophagy.

\section{Discussion}

Spermidine plays an important role in ageing during which there is a decline of its levels in different mammalian organs (Scalabrino and Ferioli 1984). The exogenous administration of spermidine promotes longevity in many model organisms including yeast, nematodes and flies, and significantly reduces age-related oxidative protein damage in mice (Eisenberg et al. 2009; Madeo et al. 2010; Morselli et al. 2011; Tirupathi et al. 2011). This increase in longevity is linked to changes in the acetylation of nuclear histones and to a transcriptional increase of different autophagy-related genes (Eisenberg et al. 2009). Furthermore, more recent studies have shown that spermidine induces autophagy through AMPK-dependent pathway which is well known to play an anti-ageing role (Morselli et al. 2011).

Spermidine plays a pivotal role in the post-translational modification of the eukaryotic initiation factor 5A (eIF5A) since it is the essential substrate for the protein hypusination (Huang et al. 2007). Under physiological conditions eIF5A is constitutively hypusinated, but its activity and subcellular localization can be conditioned by reversible acetylation (Lee et al. 2009; Ishfaq et al. 2012). PCAF is the major cellular acetyltransferase of eIF5A, and HDAC6 and SIRT2 are its major deacetylases (Ishfaq et al. 2012). Inhibition of the deacetylases or impaired hypusination increased acetylation of eIF5A, leading to its nuclear accumulation (Ishfaq et al. 2012). Considering that the mechanism by which spermidine induces autophagy is not yet well elucidated, we asked whether hypusinated eIF5A can be the link in that process.
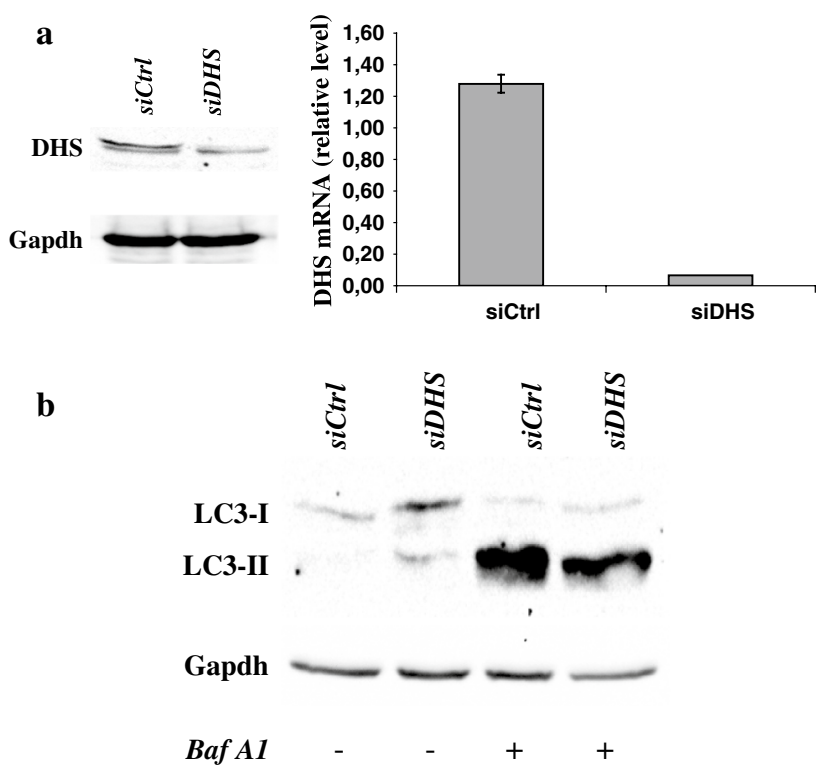

c
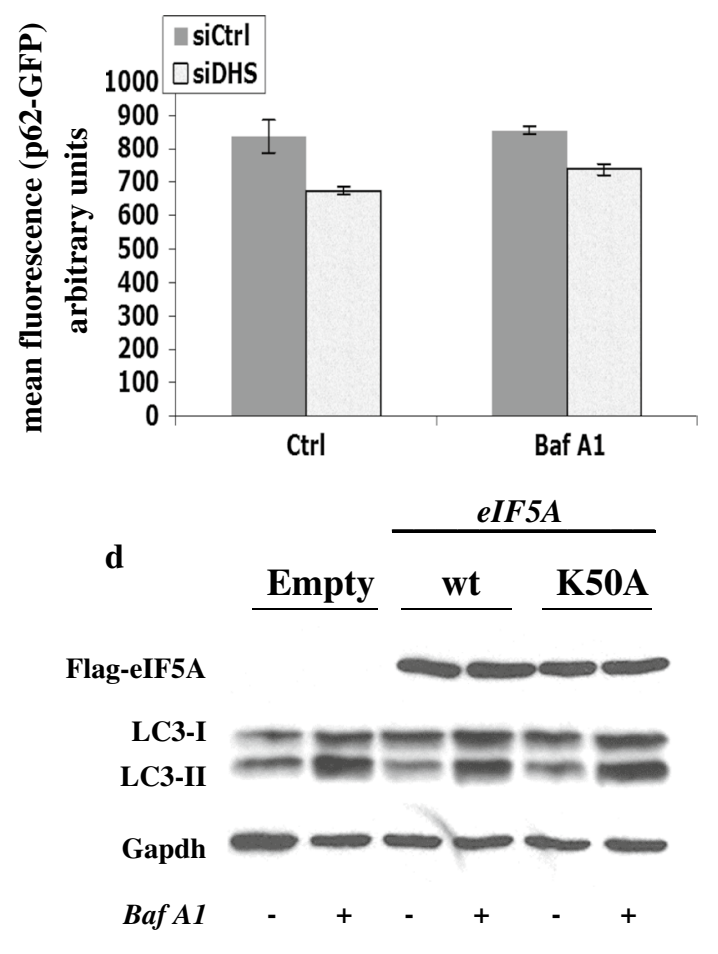

In this study, we analyzed the influence of eIF5A in the process of autophagy induced by starvation using several approaches: first, we blocked the conversion of native eIF5A into mature hypusinated protein by GC7 (N1-guanyl-1,7-diamineoheptane), or we used siRNA interference for knocking down the expression of native eIF5A and DHS or we over-expressed the native eIF5A or the mutated one in the hypusination site. Surprisingly, our results revealed not only that the GC7 alone can deregulate the basal autophagy, but also that this action is independent of eIF5A activity. 
GC7 is usually used to block the first step of hypusination of eIF5A which leads to the accumulation of the native protein (Landau et al. 2010). Interestingly, the treatment with GC7 displays an anticancer effect in various tumours such as neuroblastoma, erythroleukaemia and melanoma (Shi et al. 1996; Chen et al. 1996; Lee et al. 2002; Jasiulionis et al. 2007). Lee et al. (2009) demonstrated that GC7 inhibits growth and differentiation in oral cancer and immortalized keratinocytes by inducing apoptosis through the mitochondrial and the AMPK pathways (Lee et al. 2009). The results reported in this study are the first evidence that GC7 induces autophagy in 2fTGH cell line.

These findings are interesting considering the complex role of autophagy in cancer initiation and progression. We expected to find a block in autophagy induction in the presence of the eIF5A-hypusination inhibitor GC7; surprisingly, by contrast, we revealed an off-target effect of the drug. In fact, the ablation of both the eIF5A itself or the enzyme mediating its hypusination, DHS, resulted in no appreciable change in the autophagic flux, thus confirming that the marked pro-autophagic effect of GC7 is not mediated by the hypusine pathway. In line with this conclusion, we also demonstrate that the over-expression of both wild type or K50A mutant eIF5A, in which the hypusination site (Lys50) has been replaced by an alanine, did not affect the basal autophagy in $2 \mathrm{~F}$ cells confirming that the immature form of eIF5A (with no hypusine modification) is unrelated to the autophagic process.

In contrast to our initial hypothesis, collectively these data suggest that mature eIF5A (hypusinated form) is not involved in the autophagic pathway and that the inhibitor of deoxyhypusine synthase, GC7, has an off-target effect resulting in autophagy induction. Future studies should clarify by which mechanism GC7 is able to promote autophagy. In keeping with this assumption it is interesting to note that hypusine of the eIF-5A chain functions as an acyl acceptor substrate for transglutaminases (Beninati et al. 1995). Considering the structural similarity between hypusine molecule and GC7, it would be interesting to study whether this polyamine analogue can act as a substrate of Type 2 transglutaminase transamidating activity which has been shown to play an important role in the recruitment of ubiquitinated proteins into the autophagosomes (D'Eletto et al. 2009). The GC7 off-target effect we demonstrated in this study is particularly relevant considering that this drug has been proposed as an important candidate for the therapy of cancer, diabetes and HIV infection. Indeed, eIF5A plays an important role in protein translation since disruption of the hypusination process by GC7 has been shown to inhibit the growth of many cancer cell types as well as endothelial cells (Lee et al. 2009, 2010; Caraglia et al. 2003). For example, in hepatocellular carcinoma, over-expression of eIF5A2 was reported to be associated with tumour features that indicate poor prognosis, such as the presence of tumour metastasis and venous infiltration (Lee et al. 2010). Furthermore, the clinical drugs ciclopirox and deferiprone, by inhibiting eIF5A hypusination, impair the transcription of the HIV-1 promoters and decrease HIV-1 gene expression (Hoque et al. 2009). Based on the considerable therapeutic interest in eIF5A as a selective target for drug development through inhibition of hypusination, the GC7 off-target effect described in this study acquires particular relevance and should be taken into full consideration for the use of GC7 in clinical trials.

Acknowledgments This work was supported by grants from MIUR (PRIN 2012), AIRC (MFAG 11743 to MC; IG 2011 N.11409 to MP), FILAS, the Ministry of Health of Italy "Ricerca Corrente" and "Ricerca Finalizzata". The support of the EU Grant "Transpath" Marie Curie project to MP is also acknowledged. We thank Prof. Myung Hee Park from National Institute of Dental and Craniofacial Research "NIDCR" Bethesda, MD who kindly provided the p3XFLAG-CMV-10.1 plasmid encoding for human eIF5A-1; Alessandro Lentini for his graphical support and Maria Concetta Dell'Acqua for her technical assistance.

Conflict of interest The authors have no conflicts of interest to disclose.

\section{References}

Beninati S, Nicolini L, Jakus J, Passeggio A, Abbruzzese A (1995) Identification of a substrate site for transglutaminases on the human protein synthesis initiation factor $5 \mathrm{~A}$. Biochem $\mathrm{J}$ 305(3):725-728

Benne R, Hershey JW (1978) The mechanism of action of protein synthesis initiation factors from rabbit reticulocytes. J Biol Chem 253:3078-3087

Caraglia M, Marra M, Giuberti G, D’Alessandro AM, Baldi A, Tassone P, Venuta S, Tagliaferri P, Abbruzzese A (2003) The eukaryotic initiation factor $5 \mathrm{~A}$ is involved in the regulation of proliferation and apoptosis induced by interferon-alpha and EGF in human cancer cells. J Biochem (Tokyo) 133:757-765

Caraglia M, Park MH, Wolff EC, Marra M, Abbruzzese A (2013) eIF5A isoforms and cancer: two brothers for two functions? Amino Acids 44(1):103-109

Clement PM, Johansson HE, Wolff EC, Park MH (2006) Differential expression of eIF5A-1 and eIF5A-2 in human cancer cells. FEBS J 273(6):1102-1114

Cooper HL, Park MH, Folk JE (1982) Posttranslational formation of hypusine in a single major protein occurs generally in growing cells and is associated with activation of lymphocyte growth. Cell 29:791-797

D’Eletto M, Farrace MG, Falasca L, Reali V, Oliverio S, Melino G, Griffin M, Fimia GM, Piacentini M (2009) Transglutaminase 2 is involved in autophagosome maturation. Autophagy 5:1145-1154

Eisenberg T, Knauer H, Schauer A, Buttner S, Ruckenstuhl C, Carmona-Gutierrez D, Ring J, Schroeder S, Magnes C, Antonacci L, Fussi H, Deszcz L, Hartl R, Schraml E, Criollo A, Megalou E, Weiskopf D, Laun P, Heeren G, Breitenbach M, GrubeckLoebenstein B, Fahrenkrog B, FroÅNhlich KU, Sinner F, Tavernarakis N, Minois N, Kroemer G, Madeo F (2009) Induction of autophagy by spermidine promotes longevity. Nat Cell Biol 11:1305-1314 
Fimia GM, Piacentini M (2010) Regulation of autophagy in mammals and its interplay with apoptosis. Cell Mol Life Sci 67:1581-1588

Gerner EW, Meyskens FL Jr (2004) Polyamines and cancer: old molecules, new understanding. Nat Rev Cancer 4(10):781-792

Guan XY, Fung JM, Ma NF, Lau SH, Tai LS, Xie D, Zhang Y, Hu L, Wu QL, Fang Y et al (2004) Oncogenic role of eIF-5A2 in the development of ovarian cancer. Cancer Res 64:4197-4200

Hill DS, Martin S, Armstrong JL, Flockhart R, Tonison JJ, Simpson DG, Birch-Machin MA, Redfern CP, Lovat PE (2009) Combining the endoplasmic reticulum stress-inducing agents bortezomib and fenretinide as a novel therapeutic strategy for metastatic melanoma. Clin Cancer Res 15(4):1192-1198

Hoque M, Hanauske-Abel HM, Palumbo P, Saxena D, D'Alliessi Gandolfi D, Park MH, Pe'ery T, Mathews MB (2009) Inhibition of HIV-1 gene expression by Ciclopirox and Deferiprone, drugs that prevent hypusination of eukaryotic initiation factor 5A. Retrovirology 6:90. doi:10.1186/1742-4690-6-90

Huang Y, Higginson DS, Hester L, Park MH, Snyder SH (2007) Neuronal growth and survival mediated by eIF5A, a polyaminemodified translation initiation factor. Proc Natl Acad Sci USA 104:4194-4199

Igarashi K, Kashiwagi K (2010) Modulation of cellular function by polyamines. Int J Biochem Cell Biol 42(1):39-51

Jasiulionis MG, Luchessi AD, Moreira AG, Souza PP, Suenaga AP, Correa M, Costa CA, Curi R, Costa-Neto CM (2007) Inhibition of eukaryotic translation initiation factor 5A (eIF5A) hypusination impairs melanoma growth. Cell Biochem Funct 25:109-114

Jenkins ZA, Haag PG, Johansson HE (2001) Human eIF5A2 on chromosome $3 \mathrm{q} 25-\mathrm{q} 27$ is a phylogenetically conserved vertebrate variant of eukaryotic translation initiation factor $5 \mathrm{~A}$ with tissuespecific expression. Genomics 71:101-109

Kaiser A (2012) Translational control of eIF5A in various diseases. Amino Acids 42(2-3):679-684

Landau G, Bercovich Z, Park MH, Kahana C (2010) The role of polyamines in supporting growth of mammalian cells is mediated through their requirement for translation initiation and elongation. J Biol Chem 285:12474-12481

Lee YB, Folk JE (1998) Branched-chain and unsaturated 1,7-diaminoheptane derivatives as deoxyhypusine synthase inhibitors. Bioorg Med Chem 6(3):253-270

Lee Y, Kim HK, Park HE, Park MH, Joe YA (2002) Effect of N1-guanyl-1,7 diaminoheptane, an inhibitor of deoxyhypusine synthase, on endothelial cell growth, differentiation and apoptosis. Mol Cell Biochem 237(1-2):69-76

Lee SK, Lee J, Lee SI, Bae WJ, Lee YM, Park JS, Lee SK, Park SJ, Min SK, Kim EC (2009) N1-guanyl-1,7,-diamineoheptane, an inhibitor of deoxyhypusine synthase, suppresses differentiation and induces apoptosis via mitochondrial and AMPK pathways in immortalized and malignant human oral keratinocytes. J Oral Pathol Med 38:792-800

Lee NP, Tsang FH, Shek FH, Mao M, Dai H, Zhang C, Dong S, Guan XY, Poon RT, Luk JM (2010) Prognostic significance and therapeutic potential of eukaryotic translation initiation factor 5A (eIF5A) in hepatocellular carcinoma. Int $\mathrm{J}$ Cancer 127(4):968-976

Liu YP, Nemeroff M, Yan YP, Chen KY (1997) Interaction of eukaryotic initiation factor 5A with the human immunodeficiency virus type $1 \mathrm{Rev}$ response element RNA and U6 snRNA requires deoxyhypusine or hypusine modification. Biol Signals 6:166-174

Madeo F, Eisenberg T, Büttner S, Ruckenstuhl C, Kroemer G (2010) Spermidine: a novel autophagy inducer and longevity elixir. Autophagy 6(1):160-162

Maier B, Ogihara T, Trace AP, Tersey SA, Robbins RD, Chakrabarti SK, Nunemaker CS, Stull ND, Taylor CA, Thompson JE, Dondero RS, Lewis EC, Dinarello CA, Nadler JL, Mirmira RG (2010) The unique hypusine modification of eIF5A promotes islet $\mathrm{b}$ cell inflammation and dysfunction in mice. J Clin Invest 120:2156-2170

Mandal S, Mandal A, Johansson HE, Orialo AV, Park MH (2013) Depletion of cellular polyamines, spermidine and spermine, causes a total arrest in translation and growth in mammalians cells. PNAS 110(6):2169-2174

Moore CC, Martin EN, Lee G, Taylor C, Dondero R et al (2008) Eukaryotic translation initiation factor $5 \mathrm{~A}$ small interference RNA-liposome complexes reduce inflammation and increase survival in murine models of severe sepsis and acute lung injury. J Infect Dis 198(9):1407-1414

Morselli E, Marino G, Bennetzen MV, Eisenberg T, Megalou E, Schroeder S, Cabrera S, BeÅLnit P, Rustin P, Criollo A, Kepp O, Galluzzi L, Shen S, Malik SA, Maiuri MC, Horio Y, LoÅLpezOtiÅLn C, Andersen JS, Tavernarakis N, Madeo F, Kroemer G (2011) Spermidine and resveratrol induce autophagy by distinct pathways converging on the acetylproteome. J Cell Biol 192:615-629

Pagliarini V, Wirawan E, Romagnoli A, Ciccosanti F, Lisi G, Lippens S, Cecconi F, Fimia GM, Vandenabeele P, Corazzari M, Piacentini M (2012) Proteolysis of Ambral during apoptosis has a role in the inhibition of the autophagic pro-survival response. Cell Death Differ 19(9): 1495-1504

Park MH (2006) The post-translational synthesis of a polyaminederived amino acid, hypusine, in the eukaryotic translation initiation factor 5A (eIF5A). J Biochem (Tokyo) 139:161-169

Park MH, Wolff EC, Folk JE (1993) Hypusine: its post-translational formation in eukaryotic initiation factor $5 \mathrm{~A}$ and its potential role in cellular regulation. BioFactors 4:95-104

Park MH, Nishimura K, Zanelli CF, Valentini SR (2010) Functional significance of eIF5A and its hypusine modification in eukaryotes. Amino Acids 38:491-500

Patel PH, Costa-Mattioli M, Schulze KL, Bellen HJ (2009) The Drosophila deoxyhypusine hydroxylase homologue nero and its target eIF5A are required for cell gro wth and the regulation of autophagy. J Cell Biol 185:1181-1194

Pegg AE (2009) Mammalian polyamine metabolism and function. IUBMB Life 61(9):880-894

Saini P, Eyler DE, Green R, Dever TE (2009) Hypusine-containing protein eIF-5A promotes translation elongation. Nature 459:118121. doi: $10.1038 /$ nature 08034

Scalabrino G, Ferioli ME (1984) Polyamines in mammalian ageing: an oncological problem, too? A review. Mech Ageing Dev 26:149-164

Schnier J, Schwelberger HG, Smit-McBride Z, Kang HA, Hershey JW (1991) Translation initiation factor 5A and its hypusine modification are essential for cell viability in the yeast Saccharomyces cerevisiae. Mol Cell Biol 11:3105-3114

Shi XP, Yin KC, Ahem J, Davis LJ, Stern AM, Waxman L (1996) Effects of N 1-guanyl-1,7-diaminoheptane, an inhibitor of deoxyhypusine synthase, on the growth of tumorigenic cell lines in culture. Biochim Biophys Acta 1310(1):119-126

Shiba T, Mizote H, Kaneko T, Nakajima T, Kakimoto Y (1971) Hypusine, a new amino acid occurring in bovine brain. Isolation and structural determination. Biochim Biophys Acta 244:523-531

Sun Z, Cheng Z, Taylor CA, McConkey BJ, Thompson JE (2010) Apoptosis induction by eIF5A1 involves activation of the intrinsic mitochondrial pathway. J Cell Physiol 223:798-809

Taylor CA, Sun Z, Cliche DO, Ming H, Eshaque B, Jin S, Hopkins MT, Thai B, Thompson JE (2007) Eukaryotic translation initiation factor $5 \mathrm{~A}$ induces apoptosis in colon cancer cells and associates with the nucleus in response to tumour necrosis factor alpha signalling. Exp Cell Res 313:437-449

Taylor CA, Liu Z, Tang TC, Zheng Q, Francis S, Wang TW, Ye B, Lust JA, Dondero R, Thompson JE (2012) Modulation of eIF5A expression using SNS01 nanoparticles inhibits NF-kB activity 
and tumor growth in murine models of multiple myeloma. Mol Ther 20(7):1305-1314

Taylor CA, Zheng Q, Liu Z, Thompson JE (2013) Role of p38 and JNK MAPK signaling pathways and tumor suppressor p53 on induction of apoptosis in response to Ad-eIF5A1 in A549 lung cancer cells. Mol Cancer 12:35. doi:10.1186/1476-4598-12-35

Tirupathi Pichiah PB, Suriyakalaa U, Kamalakkannan S, Kokilavani P, Kalaiselvi S, SankarGanesh D, Gowri J, Archunan G,
Youn-Soo Cha, Achiraman S (2011) Spermidine may decrease ER stress in pancreatic beta cells and may reduce apoptosis via activating AMPK dependent autophagy pathway. Med Hypotheses 77:677-679

Xie Z, Klionsky DJ (2007) Autophagosome formation: core machinery and adaptations. Nat Cell Biol 9:1102-1109

Zuk D, Jacobson A (1998) A single amino acid substitution in yeast eIF5A results in mRNA stabilization. EMBO J 17:2914-2925 